\title{
Comparison between Conventional OCDMA and Subcarrier Multiplexing SAC OCDMA System Based on Single Photodiode Detection
}

\author{
N. A. A Ahmad, M. N Junita, Syed Alwi Aljunid, Mohd Rashidi Che Beson, Rosdisham Endut \\ Advanced Communication Engineering, \\ Centre of Excellence, \\ School of Computer and Communication Engineering, \\ Universiti Malaysia Perlis, \\ Arau, 01000, Perlis \\ Malaysia. \\ n.amirahamniahmad@gmail.com
}

\begin{abstract}
This paper demonstrates the comparison between conventional OCDMA system and subcarrier multiplexing (SCM) SAC-OCDMA system by applying Recursive Combinatorial (RC) code based on single photodiode detection (SPD). SPD is used in the receiver part to reduce the effect of multiple access interference (MAI) which contributes as a dominant noise in incoherent SAC-OCDMA systems. From this analysis, the performance of SCM OCDMA network could be improved by using lower data rates and higher received power. The hybrid SCM OCDMA system shows better performance compare to conventional OCDMA system although the number of users involved is very high. This is because, for hybrid SCM OCDMA system, the number of users can be increased by increasing the number of subcarriers without affect the number of code length and optical codes. Increasing the number of subcarriers will enhance the power consumption by applying hybrid SCM system in OCDMA compared to the conventional OCDMA system. This is because increasing the number of users for hybrid SCM system does not affects the number of code length and the number of optical codes but only increase the number of subcarriers. Thus, hybrid SCM OCDMA system has to increase spectral efficiency and produce better performance compared to conventional of OCDMA system.
\end{abstract}

\section{Introduction}

Generally, there are three types of multiplexing techniques in optical system such as Optical code division multiple access (OCDMA), Wavelength Division Multiplexing (WDM) and optical Time Division Multiplexing (TDM). OCDMA technique is a method of sharing the bandwidth of optical fiber among the number of users in the local area network [1] meanwhile WDM technique and TDM technique are applicable for long-haul networks. In addition, both of TDMA and WDMA techniques required deterministic wavelength assignment and strict synchronous time-slot control, respectively[2]. Besides that, OCDMA becomes attention because of their benefits such as it capable to uphold asynchronous access network, dynamic bandwidth assignment, all optical processing, and the ability to support multimedia services. These benefits are too important for implementation of local area network.

Basically, detection is a vital part of the system as the design of transmitter and receivers depends on the type of detection. The detections are divided by two types which are coherent and incoherent [3] but mostly system applied incoherent detection. This is because incoherent detection has numerous benefits compared to coherent. For instance, the hardware complexity of incoherent detection is less rather than coherent detection. Besides that, the phase information knowledge is needed when coherent detection sending the signal while for incoherent detection does not need the phase information knowledge. In addition, for coding operation, coherent detection performed in bipolar approach meanwhile incoherent detection performed in unipolar approach [4]. Coherent technique is difficult to apply compared to incoherent technique. Thus, in this research, we applied incoherent detection.

However, the cross correlation function is always generated in the incoherent code words which give rise to the multiple access interference (MAI) in the system. Then, in order to reduce the effects of MAI in the OCDMA system, the detection technique is proposed. There are many types of detection techniques but the 
well-known detection techniques are AND subtraction detection technique, direct detection technique, spectral direct detection technique (SDD), single photodiode detection technique and NAND subtraction technique. In this paper, we applied SPD technique to reduce the effects of MAI in the hybrid SCM system.

The effects of MAI can limit the performance of hybrid SCM OCDMA system especially when employing large number of users. So, to reduce the effects of MAI, SAC-OCDMA system needs to employ code sequence with fixed in-phase cross correlation. SAC-OCDMA systems are receiving more attention because of their ability to completely reduce multi access interference. There are numerous of code families have been developed for SAC-OCDMA such as modified quadratic congruence codes, M-sequence codes, double weight codes, etc but in this word we applied recursive combinatorial code. A part from that, the SCM scheme is capable of improving the channel data rate of OCDMA systems. This hybrid scheme is proposed for the benefit of combining the advantages of both schemes. The use of hybrid Radio Frequency technique over SCMspectral amplitude coding (SAC) Optical Code Division Multiple Access (OCDMA) is proposed to carry the high data over fiber link and to increase the number of users. This enables the reduction of Multi Access Interference (MAl) with high data rate, leading to the increase in the number of active users. Consequently, the hybrid system is robust against interference and is much more spectrally efficient.

We carried out this research by using a hybrid SCM technique by employing SAC OCDMA code sequences. By employing SCM technique, it will increase the spectral efficiency of the OCDMA system. In addition, the number of users can be increase by increasing the number subcarrier without effects the number of code length and number of SAC-OCDMA codes.

\section{Recursive Combinatorial Code}

Recursive Combinatorial (RC) code was introduced and developed by M. N Junita [5] in 2004. The advantages of $\mathrm{RC}$ codes are, it has minimum cross correlation, which is one and minimum code lengths compare to other codes. Besides that, $\mathrm{RC}$ code is flexibility in choosing code parameter. It can be shown when adding the number of users in hybrid system, it will not disturb the code words given for existing users, since new code sequence for additional users are added at the next available bandwidth. A part from that, increasing the number of weight will not affect other parameter such as the number of users, K. Thus, it will not affect the code sequences for every existing user; i.e. increasing the code weight does not affect the chips positioning

\section{Single Photodiode Detection (SPD)}

In OCDMA systems, the detection schemes affect the design of transmitter and receivers. The types of detection are divided by two which are coherent system and incoherent system. Incoherent system is unipolar sequence for the code sequence whereas coherent system is bipolar sequence for the code words. In our system, we applied incoherent system since the coherent system is phase sensitive, which would be practically difficult to implement in this system.

Figure 1 illustrates the implementation of SCM OCDMA structure at the transmitter part. Here, data from users are modulated into electrical signal within different specific frequencies. These RF frequencies are then combined using electrical adder before being modulated into optical signal codes via a Mach Zhender Modulator. The optical RC codes are executed by splitting the broadband optical sources into smaller wavelengths, and encoded it back in the form of codes using an encoder. Optical combiner is then used to combine the optical codes before being transmitted via a single mode fiber.

Figure 2 shows the implementation of SPD detection at the receiver. The received optical signal is decoded by the decoder, which has an identical spectral response to the intended encoder for the data to be received. The remainder of the signal from the decoder is then transmitted to the subtractive decoder (s-Decoder) to cancel out signals with mismatched interferers. The sDecoder contains only frequency bins from different interferers. The output from the s-Decoder is either zero power unit for active user or cross-correlation power unit for interferers.

After optical subtraction, the output is either code weight power unit for active user or zero power units for interferers. This implies that the interference signals are suppressed in the optical domain before the conversion of the signals to the electrical domain, as a result, the proposed SPD scheme alleviates both PIIN and MAI in the optical domain. Moreover, the two interference signals at the optical subtractor are assumed to be equal and cancel each other out. However, practically, the interference signals differ slightly at the optical subtractor and results in a small amount of optical power to reach the photodiode.

The main advantage of using the SPD is that the cancellation of the interference signals in the optical domain allows the use of only a single photodiode rather than two photodiodes as in typical subtraction detection schemes. This reduces the amount of optical-to-electrical conversion and shot noise generated at the receiver part [1].

At the photodetector, the photo diode only detects and filters the clean chips or the non-overlapping chips. Then, the effects of MAI are successfully reduced in the optical domain because there is only the required signal 
spectra are filtered. Then, there is no subtraction process in SPD technique. It is because; the photo detector only detects the clean optical pulses and converted into electrical domain. The electrically modulated signal is then passed through a Band Pass Filter (BPF) to remove the unwanted frequency. In order to remove the higher frequency, a low pass filter (LPF) is assigned. The original data signal is detected at the output of LPF using a BER analyser.

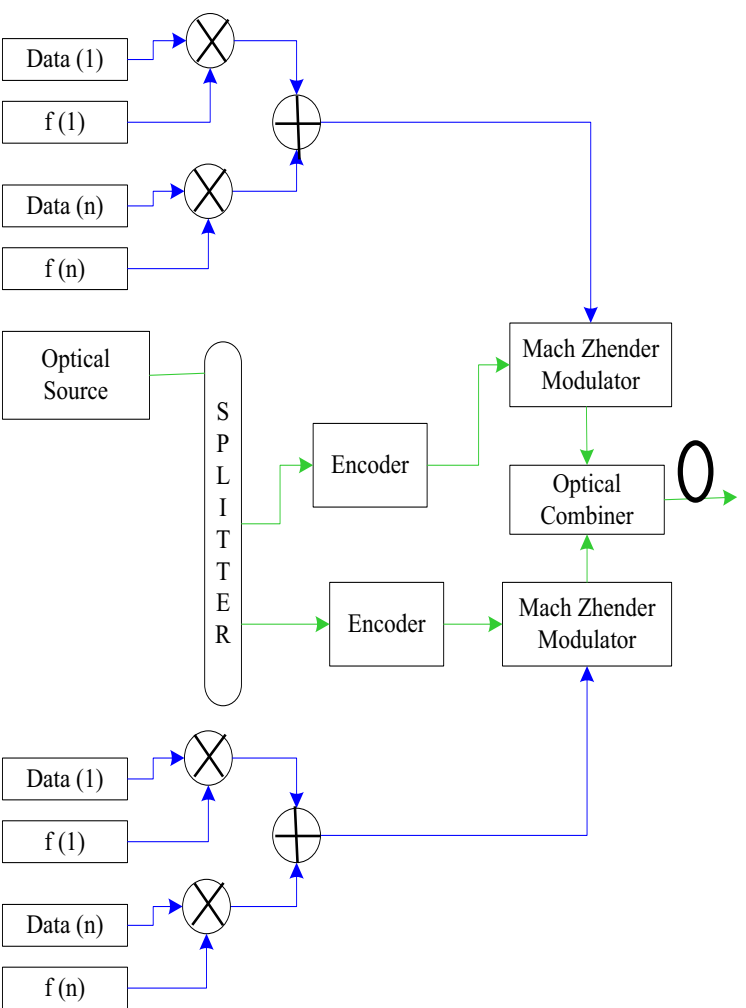

Fig. 1 : Transmitter part of hybrid SCM SAC- OCDMA system

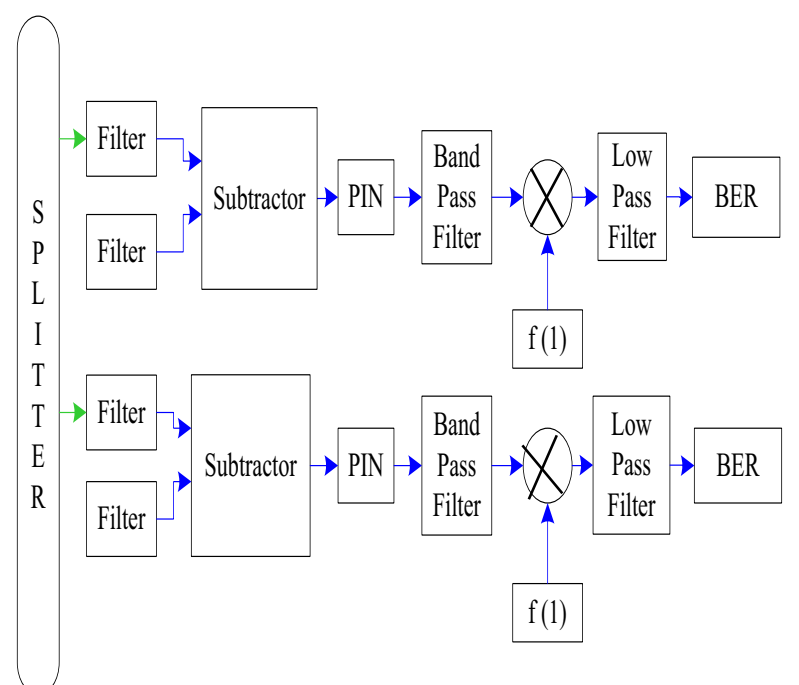

Fig. 2 : The receiver part hybrid SCM SAC-OCDMA system based on SPD detection technique

\section{Theoretical Development of Hybrid SCM OCDMA System Based on SPD Technique}

The performance of $\mathrm{RC}$ code in hybrid system can be analysed by describing the properties of RC code which are the cross correlation and auto correlation function of the code take into account. In equation $1, \mathrm{C}_{\mathrm{d}}(i)$ represents the $i$ th element of $A$ th RC code sequence. So that, the properties of RC code [5] based on SPD method can be illustrated as:

$\sum_{i=1}^{L} C_{d}(i) C_{e}(i)=\left\{\begin{array}{l}W ; \text { for } d=e \\ 1 ; \text { for } d \neq e\end{array}\right.$

Where $\mathrm{C}_{d}$ and $\mathrm{C}_{e}$ depict the $\mathrm{RC}$ code sequences, meanwhile $\mathrm{L}$ depicts the code length and $\mathrm{W}$ depicts the number of the weight. At the receiver part, the photo detector only detects and decodes the non-overlapping chips. Therefore, the total incident power during one period at the input of the photo detector PIN 1 for the SPD detection technique can be expressed by:

$\int_{0}^{\infty} G_{d d}(v) d v=$

$\int_{0}^{\infty} \frac{P_{s r}}{\Delta v} \sum_{k=1}^{K} d_{k} \sum_{i=1}^{L} C_{d}(i) C_{e}(i) u\left[\frac{\Delta v}{L}\right] d v$

(2)

$=\frac{P_{s r}}{\Delta v}\left[\frac{\Delta v}{L}\right] \sum_{k=1}^{K} d_{k} \sum_{i=1}^{l} C_{d}(i) C_{e}(i)$

$=\frac{P_{s r}}{L}(w-1)$

The photodiode current, I indicate the signal from the desired user and can be displays as:

$I=I_{d d}=\Re \int_{0}^{\infty} G_{d d}(v) d v$

Then, substitute the equation (4) in (5), the equation becomes:

$I_{d d}=\Re \frac{P_{s r}}{L}(w-1)$

Based on equation (6), (W-1) represents the power spectral density of the overlapping chips or cross correlation are subtracted at the received power signal. Thereby, the photodiode only detects and filters the PSD of the non-overlapping chips.

For the noise variances, there are three types of noises are considered which are shot noise, thermal noise and intermodulation noise while PIIN noise is neglected. The PIIN noise is neglected because it only appearance when the optical signal is incident more than one on the photo detector surface. The shot noise can be expressed as: 
$I_{\text {shot }}^{2}=2 e B I_{d d}$

$I_{\text {shot }}^{2}=2 e B \Re \frac{P_{s r}(w-1)}{L}$

The thermal noise [6] can be expressed as:

$I_{\text {thermal }}^{2}=\frac{4 \mathrm{~K}_{\mathrm{b}} \mathrm{T}_{\mathrm{N}} \mathrm{B}}{\mathrm{R}_{\mathrm{L}}}$

The inter-modulation noise [7] can be expressed as:

$I_{I M D}^{2}=\mathbf{P}_{s r}^{2} \Re^{2} \mathbf{m}_{\mathrm{n}, \mathrm{k}}^{6}\left[\frac{\mathrm{D}_{111}}{32}+\frac{\mathrm{D}_{21}}{64}\right]$

The Signal Noise Ratio or SNR equation of the hybrid SCM OCDMA using RC code using SPD method can be expressed as:

$S N R=$

$\frac{\left(\frac{\Re P_{s r}(W-1)}{L}\right)^{2} m_{n, k}^{2}}{e B \Re \frac{P_{s r(W-1)}}{L}+\frac{4 K_{b} T_{n} B}{R_{L}}+P_{s r}^{2} \Re^{2} m_{n, k}^{6}\left[\frac{D_{11}}{32}+\frac{D_{21}}{64}\right]}$

Based on equation (10), the formula to calculate the bit error rate (BER) can be written as:

$\mathrm{BER}=\frac{1}{2} \operatorname{erfc}\left(\sqrt{\frac{S N R}{8}}\right)$

Here, erf $c=\frac{2}{\sqrt{\pi}} \int_{x}^{\infty} \exp \left(-z^{2}\right) d z$

Table 1 : List of parameter used in the analysis

\begin{tabular}{|c|c|l|}
\hline Symbol & Parameter & Value \\
\hline $\boldsymbol{\eta}$ & $\begin{array}{c}\text { The efficiency of } \\
\text { photodetector quantum }\end{array}$ & 0.6 \\
\hline $\boldsymbol{v}$ & $\begin{array}{c}\text { The source of Linewidth } \\
\text { broadband }\end{array}$ & $3.75 \mathrm{THz}$ \\
\hline$\lambda_{\mathbf{0}}$ & $\begin{array}{c}\text { The wavelength of } \\
\text { operating }\end{array}$ & $1550 \mathrm{~nm}$ \\
\hline $\mathrm{B}$ & Electrical bandwidth & $80 / 331 \mathrm{MHz}$ \\
\hline $\mathrm{R}_{\mathrm{b}}$ & Data Bit Rate & $155 / 622 \mathrm{Mbps}$ \\
\hline $\mathrm{T}_{\mathrm{n}}$ & $\begin{array}{c}\text { The temperature of } \\
\text { Receiver noise }\end{array}$ & $300 \mathrm{~K}$ \\
\hline $\mathrm{R}_{\mathrm{L}}$ & $\begin{array}{c}\text { Receiver load resistor } \\
\mathrm{E}\end{array}$ Electron charge & $1030 \Omega$ \\
\hline $\mathrm{K}_{\mathrm{b}}$ & Boltzmann's constant & $\begin{array}{l}1.6 \times 10^{-19} \mathrm{C} \\
\mathrm{J} / \mathrm{K} \quad 10^{-23}\end{array}$ \\
\hline
\end{tabular}

\section{Analysis of Results and Discussion}

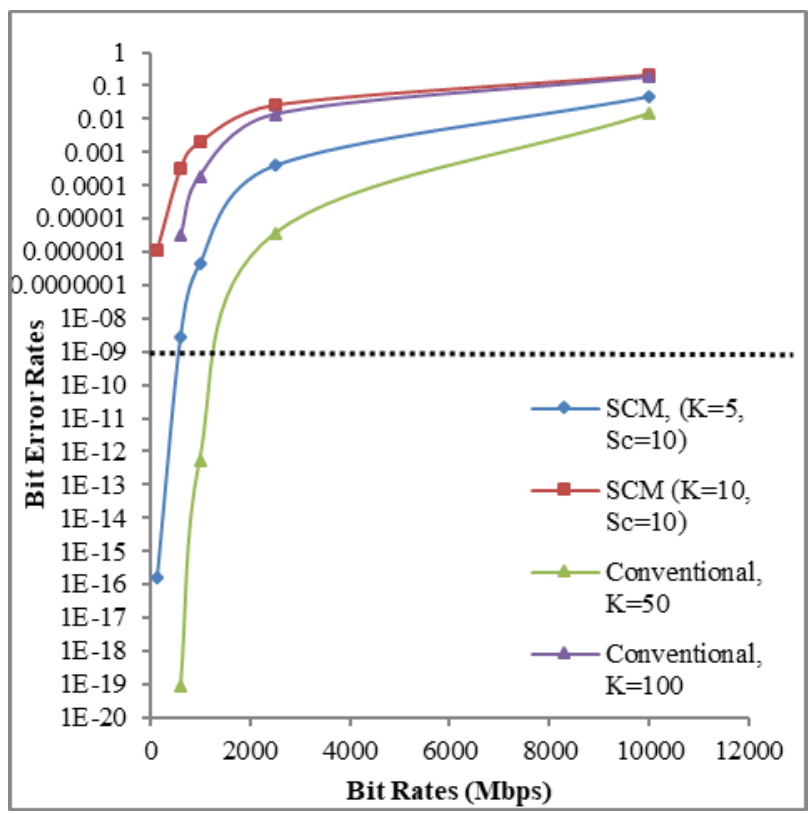

Fig. 3: The performance of bit rates versus $B E R$ at number of users, $\mathrm{K}=50$ and $\mathrm{K}=100$

From Figure 3, it indicates the comparison of performance of conventional OCDMA system and hybrid SCM SAC OCDMA system with respect to the different number of bit rates for active users equal to 50 and 100 . The bit rates are varied from $155 \mathrm{Mbps}$ to 10 Gbps. As observed in Figure 3, increasing the number of bit rate will degrades the BER performance for the both system. When higher the number of bit rate, the pulse width will be lower, thereby the signal are more sensitive to the noise. Generally, increasing the number of bit rates will generate greater electrical bandwidth and then can results in greater noise. However, hybrid SCM OCDMA system indicates better performance compared to conventional OCDMA system although the number of users involved is very high. This is because, for hybrid SCM OCDMA system, the number of users can be increase by increase the number of subcarriers without effect the number of code length and optical codes. 


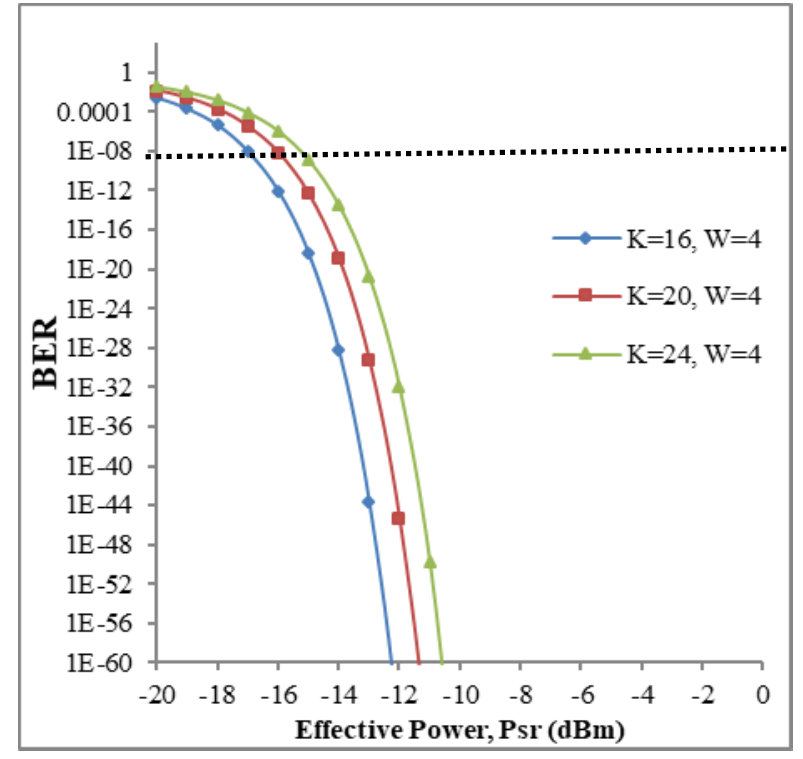

Fig. 4 : Performance of hybrid system for BER versus effective transmitted power, Psr for conventional OCDMA system.

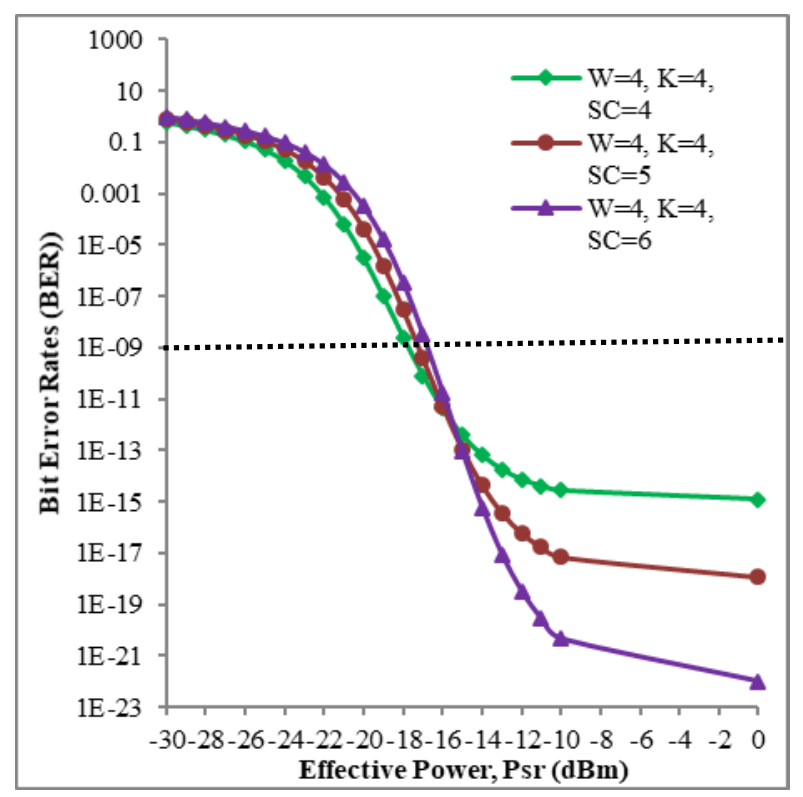

Fig. 5 : The performance of hybrid SCM OCDMA system with respect to effective power at the different number of subcarriers.

From Figure 4 and Figure 5, it depicts the effect of effective power at the photo detector versus BER. Figure 4 presents the effects of received power with varying the number of optical channel meanwhile Figure 5 indicates the effects of received power with varying the number of subcarriers. From Figure 4 and 5, it can be conclude that, the performance of BER value degrades as the number of received power decrease. The BER can degrade by some order of magnitude eventhough a tiny fall happened in the optical signal power.
Based on Figure 4, it demonstrates the performance of conventional OCDMA system with SPD detection. At the threshold BER value of $10^{-9}$, the effective power for 16 optical is about $-17 \mathrm{dBm}$, for 20 optical is approximately $-16 \mathrm{dBm}$ and for 24 optical is about -14 $\mathrm{dBm}$. So, the power different for conventional OCDMA system in order to get from 16 channels to 24 channels is about $3 \mathrm{dBm}$

Besides that, Figure 5 demonstrates hybrid SCM OCDMA system by varying the number of subcarriers and constant the number of channels. For four number of subcarriers channel, the received power is about -19 $\mathrm{dBm}$, meanwhile for five subcarriers is about $-18 \mathrm{dBm}$, for five subcarrier channel is about $-17 \mathrm{dBm}$ and for six subcarriers, the received power is about $-16 \mathrm{dBm}$ respectively. So, the power different is about $2 \mathrm{dBm}$ in order to get from 16 channel to 24 channels by utilized hybrid system. From this analysis, it can be conclude that the power range for Figure 5 is smaller than power range in Figure 4. Therefore, it is clearly presents, increasing the number of subcarriers will enhanced the power consumption by applying hybrid SCM system in OCDMA compared to the conventional all optical system in OCDMA. This is because, increasing the number of users for hybrid SCM system does not effects the number of code length and the number of optical codes but only increase the number of subcarriers. Thus, hybrid SCM OCDMA system has increase spectral efficiency compared to conventional of OCDMA system.

\section{CONCLUSION}

This paper demonstrates the comparison of conventional OCDMA system between hybrids SCM OCDMA system by applying SPD technique. The implementation of SPD technique can evaluate the performance of hybrid SCM system by employing Recursive Combinatorial code (RC). The results revealed that, it will get better performances if the number of bit rates is lower and the number of received power is higher. Besides that, hybrid SCM OCDMA system shows good performance compare to conventional OCDMA system. As the bit rates increase, it will decrease the bandwidth of the system, but for hybrid SCM OCDMA system, it still shows better performance compare to conventional OCDMA system. For the receives power, the power consumption for hybrid SCM OCDMA system also lower compared to conventional OCDMA system. This is because hybrid SCM OCDMA system can supports large number of users by increasing the number of subcarrier without effects the number of code length and optical codes. In addition, its bandwidth becomes more efficient. Thus, it can be conclude that, hybrid SCM OCDMA system shows better performance compared to conventional OCDMA system. 


\section{ACKNOWLEDGEMENT}

The authors would like to thank the Ministry of Higher Education (MOHE) Malaysia for the funding under the Fundamental Research Grant Scheme (FRGS).

\section{REFERENCES}

[1] S. G. Abdulqader, S. A. Aljunid, H. M. R. Alkhafaji, and H. A. Fadhil, "Enhanced performance of SAC-OCDMA system based on SPD detection utilizing EDFA for access networks," J. Commun., vol. 9, no. 2, pp. 99106, 2014.

[2] N. A. M. Ariff, A. Abdullah, and M. F. Nasrudin, "Advanced Computer and Communication Engineering Technology," Lect. Notes Electr. Eng., vol. 362, pp. 1209-1217, 2016.

[3] I. Rao and D. Rana, "Comparison of Different Detection Techniques Based on Enhanced Double Weight Code in Optical Code Division Multiple Access System," pp. 126-132, 2015.

[4] R. Sahbudin, S. Aljunid, M. Abdullah, M. B. A. Samad, M. Mahdi, and M. Ismail, "Comparative Performance of Hybrid SCM SAC- OCDMA System Using Complementary and AND Subtraction Detection Techniques," Iajit, vol. 5, no. 1, pp. 61-65, 2006.

[5] J. M. Nordin, S. A. Aljunid, A. M. Safar, A. R. Arief, R. A. Rahim, and R. B. Ahmad, "Design Construction and Performance of Recursive Combinatorial Code for Enhancement in Optical Code Division Multiple Access Systems," Aust. J. Basic Appl. Sci., vol. 8, no. 1, pp. 343-350, 2014.

[6] Somaya A. Abd E, Mottaleb, Heba A. Fayed, Ahmed Abd El Aziz, and Moustafa H.Aly, "SAC- OCDMA System Using Different Detection Techniques," IOSR J. Electron. Commun. Eng., vol. 9, no. 2, pp. 55-60, 2014.

[7] T. H. Abd, S. A. Aljunid, H. A. Fadhil, I. F. Radhi, R. B. Ahmad, and M. A. Rashid, "Performance improvement of hybrid SCM SAC-OCDMA networks using multi-diagonal csode," Sci. Res. Essays, vol. 7, no. 11, pp. 1262-1272, 2012. 